CERN-TH-7337/94

FTUAM-94/15

June, 1994

hep-th/9406206

\title{
A Canonical Approach to Duality Transformations
}

\author{
Enrique Álvarez 凹, Luis Álvarez-Gaumé and Yolanda Lozano ${ }^{1}$ \\ Theory Division CERN \\ 1211 Geneva 23 \\ Switzerland
}

\begin{abstract}
We show that Buscher's abelian duality transformation rules can be recovered in a very simple way by performing a canonical transformation first suggested by Giveon, Rabinovici and Veneziano. We explore the properties of this transformation, and also discuss some aspects of non-abelian duality.
\end{abstract}

\footnotetext{
${ }^{1}$ On leave of absence from: Departamento de Física Teórica, Universidad Autónoma de Madrid, 28049 Madrid, Spain
} 


\section{Introduction}

(Target space) duality, the generalization of the well-known $R$-duality in toroidal compactifications in String Theory [1], remains a promising avenue to further understand the underlying symmetries of String Theory.

The most powerful description of this symmetry was first introduced by Buscher [2], and then further elaborated upon by many others [4] [5] [6] [7] [8]. It starts with the sigmamodel formulation of the corresponding conformal field theory ("string vacuum"), and it works when the target space metric has at least one continuous isometry. Even in this case, however, the procedure looks unnecessarily complicated: the isometry is gauged, the (non propagating) gauge fields are constrained to be trivial, and the Lagrange multipliers themselves are promoted to the rank of new coordinates once the gaussian integration over the gauge fields is performed. One suspects that all those complicated intermediate steps could be avoided, and that it should be possible to pass directly from the original to the dual theory.

Some suggestions have indeed been made in the literature pointing (at least in the simplified situation where all backgrounds are constant or dependent only on t) towards an understanding of duality as particular instances of canonical transformations [9].

Our aim in this paper is to follow this lead. We shall find that it works well when the isometry is abelian, laying the theory on a simpler setting than before, namely as a (privileged) subgroup of the whole group of (non-anomalous, that is implementable in Quantum Field Theory [10], [11] [12]) canonical transformations on the phase space of the theory.

We shall be able to perform the transformation starting from arbitrary coordinates (and writing the dual manifold in arbitrary coordinates as well) and, besides, we will also follow explicitly the transformations of the currents of both the initial and the dual model. In particular in the case of WZW models [13] it becomes rather simple to prove that the full duality group is given by $A u t(G)_{L} \times A u t(G)_{R}$, where $L, R$ refer to the left- and right-currents on the model with group $G$, and $A u t(G)$ are the automorphisms of $\mathrm{G}$, both inner and outer. Due to the chiral conservation of the currents in this case, the canonical transformation leads to a local expression for the dual currents. In the case where the currents are not chirally conserved, then those currents associated to symmetries not commuting with the one used to perform duality become generically non-local in the dual theory and this is why they are not manifest in the dual Lagrangian [21.

It is, however, somewhat disappointing to have to report that we have not been able to understand non-abelian duality in terms of canonical transformations. Once again in the case of WZW-models, due to the presence of chirally conserved currents one can compute the dual theory in terms of a set of non-local variables introduced in [8]. This procedure unfortunately only works for theories with chiral currents, and even for that case we lack a Hamiltonian formulation of the transformation. 


\section{Abelian Duality and Canonical Transformations}

We start with the bosonic sigma model written in arbitrary coordinates on a manifold $M$ with Lagrangian

$$
L=\frac{1}{2}\left(g_{\mu \nu}+b_{\mu \nu}\right)(\phi) \partial_{+} \phi^{\mu} \partial_{-} \phi^{\nu}
$$

where $x^{ \pm}=(\tau \pm \sigma) / 2, \mu, \nu=1, \ldots, n=\operatorname{dim} M$. The corresponding Hamiltonian is

$$
H=\frac{1}{2}\left(g^{\mu \nu}\left(p_{\mu}-b_{\mu \rho} \phi^{\prime \rho}\right)\left(p_{\nu}-b_{\nu \sigma} \phi^{\prime \sigma}\right)+g_{\mu \nu} \phi^{\prime \mu} \phi^{\prime \nu}\right)
$$

where $\phi^{\prime \mu} \equiv d \phi^{\mu} / d \sigma$. We assume, moreover, that there is a Killing vector field $k^{\mu}$, $\mathcal{L}_{k} g_{\mu \nu}=0$ and $i_{k} H=-d v$ for some one-form $v$, where $\left(i_{k} H\right)_{\mu \nu} \equiv k^{\rho} H_{\rho \mu \nu}$ and $H=d b$ locally. This guarantees the existence of a particular system of coordinates ("adapted coordinates"), which we shall denote by $x^{a} \equiv\left(\theta, x^{i}\right)$, such that $\vec{k}=\partial / \partial \theta$. We shall denote the jacobian matrix by $e_{\mu}^{a} \equiv \partial x^{a} / \partial \phi^{\mu}$. In practice, this means that we have to complete the Killing vector $\vec{k} \equiv k^{\mu} \partial / \partial \phi^{\mu}$ with $(n-1)$ other commuting vectors in order to form a holonomic n-bein: $\left[\vec{k}, \overrightarrow{e_{i}}\right]=\left[\overrightarrow{e_{i}}, \overrightarrow{e_{j}}\right]=0$.

This then defines a point transformation in the original Lagrangian (2. 1) which acts on the Hamiltonian as a canonical transformation with generating function $\Phi=x^{a}(\phi) p_{a}$, which yields:

$$
\begin{array}{r}
p_{\mu}=e_{\mu}^{a} p_{a} \\
x^{a}=x^{a}(\phi)
\end{array}
$$

Once in adapted coordinates we can write the sigma model Lagrangian as

$$
L=\frac{1}{2} G\left(\dot{\theta}^{2}-\theta^{\prime}{ }^{2}\right)+\left(\dot{\theta}+\theta^{\prime}\right) J_{-}+\left(\dot{\theta}-\theta^{\prime}\right) J_{+}+V
$$

where

$$
\begin{array}{r}
G=g_{00}=k^{2} \quad V=\frac{1}{2}\left(g_{i j}+b_{i j}\right) \partial_{+} x^{i} \partial_{-} x^{j} \\
J_{-}=\frac{1}{2}\left(g_{0 i}+b_{0 i}\right) \partial_{-} x^{i} \quad J_{+}=\frac{1}{2}\left(g_{0 i}-b_{0 i}\right) \partial_{+} x^{i}
\end{array}
$$

In finding the dual with a canonical transformation we can use the Routh function with respect to $\theta$, i.e. we only apply the Legendre transformation to $(\theta, \dot{\theta})$. The canonical momentum is given by

$$
p_{\theta}=G \dot{\theta}+\left(J_{+}+J_{-}\right)
$$

and the Hamiltonian

$$
\begin{aligned}
& H=p_{\theta} \dot{\theta}-L=\frac{1}{2} G^{-1} p_{\theta}^{2}-G^{-1}\left(J_{+}+J_{-}\right) p_{\theta}+\frac{1}{2} G \theta^{\prime 2}+ \\
& +\frac{1}{2} G^{-1}\left(J_{+}+J_{-}\right)^{2}+\theta^{\prime}\left(J_{+}-J_{-}\right)-V .
\end{aligned}
$$


The Hamilton equations are:

$$
\begin{aligned}
\dot{\theta} & =\frac{\delta H}{\delta p_{\theta}}=G^{-1}\left(p_{\theta}-J_{+}-J_{-}\right) \\
\dot{p_{\theta}} & =-\frac{\delta H}{\delta \theta}=\left(G \theta^{\prime}+J_{+}-J_{-}\right)^{\prime}
\end{aligned}
$$

where the last equation is equivalent to current conservation.

The current components are:

$$
\begin{aligned}
& \mathcal{J}_{+}=\frac{1}{2} G \partial_{+} \theta+J_{+}=\frac{1}{2} p_{\theta}+\frac{1}{2} G \theta^{\prime}+\frac{J_{+}-J_{-}}{2} \\
& \mathcal{J}_{-}=\frac{1}{2} G \partial_{-} \theta+J_{-}=\frac{1}{2} p_{\theta}-\frac{1}{2} G \theta^{\prime}-\frac{J_{+}-J_{-}}{2}
\end{aligned}
$$

Then $\partial_{-} \mathcal{J}_{+}+\partial_{+} \mathcal{J}_{-}=0 \Leftrightarrow \dot{p_{\theta}}=-\delta H / \delta \theta$. The generator of the canonical transformation we choose is:

$$
F=\frac{1}{2} \int_{D, \partial D=S^{1}} d \tilde{\theta} \wedge d \theta=\frac{1}{2} \oint_{S^{1}}\left(\theta^{\prime} \tilde{\theta}-\theta \tilde{\theta}^{\prime}\right) d \sigma
$$

that is,

$$
\begin{gathered}
p_{\theta}=\frac{\delta F}{\delta \theta}=-\tilde{\theta}^{\prime} \\
p_{\tilde{\theta}}=-\frac{\delta F}{\delta \tilde{\theta}}=-\theta^{\prime}
\end{gathered}
$$

This generating functional does not receive any quantum corrections (as explained in [10]) since it is linear in $\theta$ and $\tilde{\theta}$. It $\theta$ were not an adapted coordinate to a continuous isometry, the canonical transformation would generically lead to a non-local form of the dual Hamiltonian. Since the Lagrangian and Hamiltonian in our case only depend on the time- and space-derivatives of $\theta$, there are no problems with non-locality. Below we will show how to transform wave functionals from the original to the dual theory.

The dual Hamiltonian is:

$$
\begin{array}{r}
\tilde{H}=\frac{1}{2} G^{-1} \tilde{\theta}^{\prime}+G^{-1}\left(J_{+}+J_{-}\right) \tilde{\theta}^{\prime}+ \\
\frac{1}{2} G p_{\tilde{\theta}}^{2}-\left(J_{+}-J_{-}\right) p_{\tilde{\theta}}+\frac{1}{2} G^{-1}\left(J_{+}+J_{-}\right)^{2}-V
\end{array}
$$

Since:

$$
\dot{\tilde{\theta}}=\frac{\delta \tilde{H}}{\delta p_{\tilde{\theta}}}=G p_{\tilde{\theta}}-\left(J_{+}-J_{-}\right),
$$

we can perform the inverse Legendre transform:

$$
\begin{aligned}
& \tilde{L}=\frac{1}{2} G^{-1}\left(\dot{\tilde{\theta}}^{2}-\tilde{\theta}^{\prime} 2\right)+G^{-1} J_{+}\left(\dot{\tilde{\theta}}-\tilde{\theta}^{\prime}\right) \\
& -G^{-1} J_{-}\left(\dot{\tilde{\theta}}+\tilde{\theta}^{\prime}\right)+V-2 G^{-1} J_{+} J_{-} .
\end{aligned}
$$


When translated in terms of $g_{a b}, b_{a b}$ this is nothing but Buscher's transformations?

$$
\begin{array}{ll}
\tilde{g}_{00}=1 / g_{00}, & \tilde{g}_{0 i}=-b_{0 i} / g_{00}, \quad \tilde{g}_{i j}=g_{i j}-\frac{g_{0 i} g_{0 j}-b_{0 i} b_{0 j}}{g_{00}} \\
\tilde{b}_{0 i}=-\frac{g_{0 i}}{g_{00}}, & \tilde{b}_{i j}=b_{i j}-\frac{g_{0 i} b_{0 j}-g_{0 j} b_{0 i}}{g_{00}}
\end{array}
$$

For the dual theory to be conformal invariant the dilaton must transform as $\Phi^{\prime}=\Phi-$ $\log g_{00}$ [2] [3].

Some useful information can be extracted easily in the approach of the canonical transformation:

-From the generating functional (2.10) we can learn about the multivaluedness and periods of the dual variables [6]. Since $\theta$ is periodic and in the path integral the canonical transformation is implemented by [10]:

$$
\psi_{k}[\tilde{\theta}(\sigma)]=N(k) \int \mathcal{D} \theta(\sigma) e^{i F[\tilde{\theta}, \theta(\sigma)]} \phi_{k}[\theta(\sigma)]
$$

where $N(k)$ is a normalization factor, $\phi_{k}(\theta+a)=\phi_{k}(\theta)$ implies for $\tilde{\theta}: \tilde{\theta}(\sigma+2 \pi)-\tilde{\theta}(\sigma)=$ $4 \pi / a$, which means that $\tilde{\theta}$ must live in the dual lattice of $\theta$. Note that (2.16) suffices to construct the dual Hamiltonian. It is a simple exercise to check that acting with (2.12) on the left-hand side of (2.16) and pushing the dual Hamiltonian through the integral we obtain the original Hamiltonian acting on $\phi_{k}[\theta(\sigma)]$ :

$$
\tilde{H} \psi_{k}[\tilde{\theta}(\sigma)]=N(k) \int \mathcal{D} \theta(\sigma) e^{i F[\tilde{\theta}, \theta(\sigma)]} H \phi_{k}[\theta(\sigma)]
$$

This makes the duality transformation very simple conceptually, and it also implies how it can be applied to arbitrary genus Riemann surfaces, because the state $\phi_{k}[\theta(\sigma)]$ could be the state obtained by integrating the original theory on an arbitrary Riemann surface with boundary. It is also clear that the arguments generalize straightforwardly when we have several commuting isometries.

-We can easily see that under the canonical transformation the Hamilton equations are interchanged:

$$
\begin{array}{r}
\dot{p_{\theta}}=-\frac{\delta H}{\delta \theta}=\left(G \theta^{\prime}+J_{+}-J_{-}\right)^{\prime} \rightarrow \dot{\tilde{\theta}}=G p_{\tilde{\theta}}-J_{+}+J_{-} \\
\dot{\theta}=\frac{\delta H}{\delta p_{\theta}}=G^{-1}\left(p_{\theta}-J_{+}-J_{-}\right) \rightarrow \dot{p_{\tilde{\theta}}}=\left(G^{-1}\left(\tilde{\theta}^{\prime}+J_{+}+J_{-}\right)\right)^{\prime}
\end{array}
$$

The canonical transformed current conservation law is in this case equivalent to the first Hamilton equation:

$$
\partial_{-} \mathcal{J}_{+}{ }^{c . t .}+\partial_{+} \mathcal{J}_{-}{ }^{c . t .}=0 \Leftrightarrow \dot{\tilde{\theta}}=\frac{\delta \tilde{H}}{\delta p_{\tilde{\theta}}}
$$

\footnotetext{
${ }^{2}$ The minus signs in $\tilde{g}_{0 i}$ and $\tilde{b}_{0 i}$ can be absorbed in a redefinition $\tilde{\theta} \rightarrow-\tilde{\theta}$.
} 
(where we denote the canonical transformed current by $\mathcal{J}_{ \pm}^{\text {c.t. }}$ ) whereas the dual current conservation law is equivalent to the second Hamilton equation.

-In the chiral case $J_{-}=0$ (i.e. $g_{0 i}=-b_{0 i}$ ) and $G$ is a constant, therefore we can normalize $\theta$ to set $G=1$ and :

$$
L=\frac{1}{2}\left(\dot{\theta}^{2}-\theta^{\prime}\right)+\left(\dot{\theta}-\theta^{\prime}\right) J_{+}+V
$$

The Hamiltonian is

$$
H=\frac{1}{2} p_{\theta}^{2}-J_{+} p_{\theta}+\frac{1}{2}\left(J_{+}+\theta^{\prime}\right)^{2}-V
$$

The action is invariant under $\delta \theta=\alpha\left(x^{+}\right)$, a $U(1)_{L}$ Kac-Moody symmetry. The $U(1)$ Kac-Moody algebra has the automorphism $\mathcal{J}_{+} \rightarrow-\mathcal{J}_{+}$. This is precisely the effect of the canonical transformation. The equation of motion or current conservation is:

$$
\partial_{-}\left(\partial_{+} \theta+J_{+}\right)=0
$$

$\mathcal{J}_{+}=\partial_{+} \theta+J_{+}=p_{\theta}+\theta^{\prime}$ transforms under the canonical transformation in $\mathcal{J}_{+}^{\text {c.t. }}=$ $-\tilde{\theta}^{\prime}-p_{\tilde{\theta}}=-\mathcal{J}_{+}$.

-We can also follow the transformation to the dual model of other continuous symmetries. The simplest case is as usual the WZW-model [13] which is the basic model with chiral currents. Consider for simplicity the level- $k S U(2)-W Z W$ model with action

$$
S[g]=\frac{-k}{2 \pi} \int d^{2} \sigma \operatorname{Tr}\left(g^{-1} \partial_{+} g g^{-1} \partial_{-} g\right)+\frac{k}{12 \pi} \int \operatorname{Tr}\left(g^{-1} d g\right)^{3},
$$

parametrizing $g$ in terms of Euler angles,

$$
g=e^{i \alpha \sigma_{3} / 2} e^{i \beta \sigma_{1} / 2} e^{i \gamma \sigma_{3} / 2}
$$

the left- and right-chiral currents are

$$
\mathcal{J}_{+}=\frac{k}{2 \pi} \partial_{+} g g^{-1} \quad \mathcal{J}_{-}=-\frac{k}{2 \pi} g^{-1} \partial_{-} g
$$

Explicitly for $\mathcal{J}_{+}$:

$$
\begin{aligned}
\mathcal{J}_{+}^{1} & =\frac{k}{2 \pi}\left(-\cos \alpha \sin \beta \partial_{+} \gamma+\sin \alpha \partial_{+} \beta\right) \\
\mathcal{J}_{+}^{2} & =\frac{k}{2 \pi}\left(\sin \alpha \sin \beta \partial_{+} \gamma+\cos \alpha \partial_{+} \beta\right) \\
\mathcal{J}_{+}^{3} & =\frac{k}{2 \pi}\left(\partial_{+} \alpha+\cos \beta \partial_{+} \gamma\right),
\end{aligned}
$$

and similarly for the right currents. If we perform duality with respect to $\alpha \rightarrow \alpha+$ constant we know that $\mathcal{J}_{+}^{3} \rightarrow-\mathcal{J}_{+}^{3}, \mathcal{J}_{-}^{3} \rightarrow \mathcal{J}_{-}^{3}$. For these currents it is easy to find the action of the canonical transformation because only the derivatives of $\alpha$ appear. For $\mathcal{J}_{+}^{1,2}$ there is an explicit dependence on $\alpha$ and it seems that the transform of these currents is very non-local. However due to the chiral nature of these currents, we can show now that there

\footnotetext{
${ }^{3}$ Note that the canonical transformed current does not coincide in general with the dual current.
} 
are similar chirally conserved currents in the dual model. To do this we first combine the currents in terms of root generators,

$$
\begin{aligned}
& \mathcal{J}_{+}^{(+)}=\mathcal{J}_{+}^{1}+i \mathcal{J}_{+}^{2}=e^{-i \alpha}\left(i \partial_{+} \beta-\sin \beta \partial_{+} \gamma\right)=e^{-i \alpha} j_{+}^{(+)} \\
& \mathcal{J}_{+}^{(-)}=\mathcal{J}_{+}^{1}-i \mathcal{J}_{+}^{2}=-e^{i \alpha}\left(i \partial_{+} \beta+\sin \beta \partial_{+} \gamma\right)=e^{i \alpha} j_{+}^{(-)}
\end{aligned}
$$

from chiral current conservation $\partial_{-} \mathcal{J}_{+}^{( \pm)}=0$ we obtain

$$
\partial_{-} j_{+}^{( \pm)}= \pm i \partial_{-} \alpha j_{+}^{( \pm)}
$$

in these equations only $\dot{\alpha}, \alpha^{\prime}$ appear, and after the canonical transformation we can reconstruct the dual non-abelian currents ( in the previous equations the canonical transformation amounts to the replacement $\alpha \rightarrow \tilde{\alpha}$ ) which take the same form as the original ones except that with respect to the transformed $\mathcal{J}_{+}^{3}$ the roles of positive and negative roots get exchanged. One also verifies that $\mathcal{J}_{-}^{a}$ are unaffected. This implies therefore that the effect of duality with respect to shifts of $\alpha$ is an automorphism of the current algebra amounting to performing a Weyl transformation on the left currents only while the right ones remain unmodified. This result although known [5] is much easier to derive in the Hamiltonian formalism than in the Lagrangian formalism where one must introduce external sources which carry some ambiguities. The construction for $S U(2)$ can be straightforwardly extended to other groups. This implies that for WZW-models the full duality group is $\operatorname{Aut}(G)_{L} \times \operatorname{Aut}(G)_{R}$, where $\operatorname{Aut}(G)$ is the group of automorphisms of the group $G$, including Weyl transformations and outer automorphisms. For instance if we take $S U(N)$, the transformation $J_{+} \rightarrow-J_{+}^{T}$, i.e. charge conjugation, follows from a canonical transformation of the type discussed. It suffices to take as generating functions for the canonical transformation the sum of the generating functions for each generator in the Cartan subalgebra. External automorphisms have been used recently to extend the notion of duality [18] [19] [20]. It is important to remark that the chiral conservation of the currents is crucial to guarantee the locality of the dual non-abelian currents. If the conserved current with respect to which we dualize is not chirally conserved locality is not obtained. The simplest example to verify this is the principal chiral model for $S U(2)$. Although it is not a CFT, it serves for illustrative purposes. The equations of motion for this model imply the conservation laws:

$$
\partial_{-} \mathcal{J}_{+}^{a}+\partial_{+} \mathcal{J}_{-}^{a}=0
$$

where

$$
\mathcal{J}_{ \pm}^{a}=\frac{k}{2 \pi} \partial_{ \pm} g g^{-1}
$$

If we perform duality with respect to the invariance under $\alpha$ translations we know how $\mathcal{J}_{ \pm}^{3}$ transform, since they are the currents associated to the isometry. With the canonical transformation is possible to see as well which are the other dual conserved currents. Since the dual model is only $U(1)$-invariant [6] one expects the rest of the currents to become non-local [21] if they exist at all. In terms of the root generators introduced in (2.27) the conservation laws

$$
\partial_{-} \mathcal{J}_{+}^{( \pm)}+\partial_{+} \mathcal{J}_{-}^{( \pm)}=0
$$


are expressed:

$$
\partial_{-} j_{+}^{( \pm)}+\partial_{+} j_{-}^{( \pm)} \mp i\left(\partial_{-} \alpha j_{+}^{( \pm)}+\partial_{+} \alpha j_{-}^{( \pm)}\right)=0
$$

Performing the canonical transformation we obtain that the dual conserved currents are given by:

$$
\begin{array}{r}
\tilde{\mathcal{J}}_{ \pm}^{(+)}=\exp \left(i \int d \sigma\left(\dot{\tilde{\alpha}}+\cos \beta \gamma^{\prime}\right)\right)\left(i \partial_{ \pm} \beta-\sin \beta \partial_{ \pm} \gamma\right) \\
\tilde{\mathcal{J}}_{ \pm}^{(-)}=-\exp \left(-i \int d \sigma\left(\dot{\tilde{\alpha}}+\cos \beta \gamma^{\prime}\right)\right)\left(i \partial_{ \pm} \beta+\sin \beta \partial_{ \pm} \gamma\right)
\end{array}
$$

which cannot be expressed in a local form.

The dual manifold $\tilde{M}\left(\tilde{\theta}, x^{i}\right)$ is automatically expressed in coordinates adapted to the dual Killing vector $\tilde{\vec{k}}=\partial / \partial \tilde{\theta}$. We can now perform another point transformation, with the same jacobian as before

$$
\tilde{e}_{\mu}^{a}=e_{\mu}^{a}
$$

to express the dual manifold in coordinates which are as close as possible to the original ones.

The transformations we have performed are: First a point transformation $\phi^{\mu} \rightarrow$ $\left\{\theta, x^{i}\right\}$, to go to adapted coordinates in the original manifold. Then a canonical transformation $\left\{\theta, x^{i}\right\} \rightarrow\left\{\tilde{\theta}, x^{i}\right\}$, which is the true duality transformation. And finally another point transformation $\left\{\tilde{\theta}, x^{i}\right\} \rightarrow \tilde{\phi}^{\mu}$, with the same jacobian as the first point transformation, to express the dual manifold in general coordinates.

It turns out that the composition of these three transformations can be expressed in geometrical terms using only the Killing vector $k^{\mu}, \omega_{\mu} \equiv e_{\mu}^{0}$ and the corresponding dual quantities $\mathrm{H}$.

It is then quite easy to check that the total canonical transformation to be made in (2.1) is just

$$
\begin{aligned}
& k^{\mu} p_{\mu} \rightarrow \tilde{\omega}_{\mu} \tilde{\phi}^{\prime \mu} \\
& \omega_{\mu} \phi^{\prime \mu} \rightarrow \tilde{k}^{\mu} \tilde{p}_{\mu}
\end{aligned}
$$

whose generating function is

$$
F=\frac{1}{2} \int_{D} \tilde{\omega} \wedge \omega=\frac{1}{2} \int_{D} \tilde{\omega}_{\mu} d \tilde{\phi}^{\mu} \wedge \omega_{\rho} d \phi^{\rho}
$$

\footnotetext{
${ }^{4}$ Please note that we must raise and lower indices with the dual metric, i.e. $\tilde{e}_{a \mu}=\tilde{g}_{a b} \tilde{e}_{\mu}^{b} ; \tilde{e}^{a \mu}=$ $\tilde{g}^{\mu \nu} \tilde{e}_{\nu}^{a}$, and this implies $\tilde{\omega}_{\mu}=\omega_{\mu}$, but $\tilde{\omega}^{\mu}=k^{\mu}\left(k^{2}+v^{2}\right)+\vec{e}^{\mu} \cdot v\left(\right.$ where $\left.\vec{e}^{\mu} \equiv e_{i}^{\mu}\right) ; \tilde{k}^{\mu}=k^{\mu}$, but $\tilde{k}_{\mu}=\left(\omega_{\mu}-\left(\vec{e}_{\mu} \cdot v\right)\right) / k^{2}$; we have moreover $\tilde{\omega}^{2}=k^{2}+v^{2}+g^{i j} v_{j} \omega_{i}$ and $\tilde{k}^{2}=1 / k^{2}$.

${ }^{5}$ The one-form $\omega \equiv \omega_{\mu} d \phi^{\mu}$ is dual to the Killing vector $\vec{k}: \omega(\vec{k})=1, \omega\left(\vec{e}_{i}\right)=0$; but it is of course different from $\underline{k} \equiv k_{\mu} / k^{2} d \phi^{\mu}$ (the former is an exact form, whereas the latter does not even in general satisfy Frobenius condition $\underline{k} \wedge d \underline{k}=0)$.
} 
One then easily performs the transformations in such a way that the dual metric and torsion can be expressed in geometrical terms as

$$
\begin{gathered}
\tilde{g}_{\mu \nu}=g_{\mu \nu}-\frac{1}{k^{2}}\left(k_{\mu} k_{\nu}-\left(v_{\mu}-\omega_{\mu}\right)\left(v_{\nu}-\omega_{\nu}\right)\right) \\
\tilde{g}^{\mu \nu}=g^{\mu \nu}+\frac{1}{(1+k . v)^{2}}\left[\left(k^{2}+(v-\omega)^{2}\right) k^{\mu} k^{\nu}-2(1+k . v)\left(k^{(\mu}(v-\omega)^{\nu)}\right]\right.
\end{gathered}
$$

and

$$
\tilde{b}_{\mu \nu}=b_{\mu \nu}-\frac{2}{k^{2}} k_{[\mu}(v-\omega)_{\nu]}
$$

which are the generalization of Buscher's formulae (2.15) in arbitrary coordinates, where

$$
\begin{aligned}
k_{(\mu}(v-\omega)_{\nu)} & =\frac{1}{2}\left(k_{\mu}\left(v_{\nu}-\omega_{\nu}\right)+k_{\nu}\left(v_{\mu}-\omega_{\mu}\right)\right) \\
k_{[\mu}(v-\omega)_{\nu]} & =\frac{1}{2}\left(k_{\mu}\left(v_{\nu}-\omega_{\nu}\right)-k_{\nu}\left(v_{\mu}-\omega_{\mu}\right)\right)
\end{aligned}
$$

\section{Non-abelian Duality}

In view of the simplicity of the canonical approach to abelian duality, one could be tempted to think that the corresponding generalization to the non-abelian case would not be very difficult. Unfortunately this is not the case, the reason being that there are no adapted coordinates to a set of non-commuting isometries, and therefore one is led to a non-local form of the Hamiltonian. In [8] we could carry out the non-abelian duality transformation due to the existence of left- and right-chiral currents and as a consequence of the PolyakovWiegmann [14] property satisfied by WZW-actions. Although in the intermediate steps it was necessary to introduce non-local variables (a convenient representation of the auxiliary variables setting the non-abelian gauge field strength to zero), the final result led to a local action in the new variables as a result of the special properties of WZW-models mentioned. The computations could be carried out exactly until the end to evaluate the form of the effective action in terms of the auxiliary variables needed in the construction of non-abelian duals. Although a glance to the action could lead us to think that the non-abelian dual of a WZW-model with group $G$ with respect to a non-anomalous subgroup $H$ is locally a coset theory $G / H$ times a WZW-theory for the subgroup $H$, a careful analysis of the BRST constraints that appear in the construction together with a computation of the duality transformation for toroidal world-sheets show that the model is self-dual. We have so far been unable to express these functional integral manipulations in a Hamiltonian setting as in previous section. We should mention however that the use of non-local variables introduced in [8] in cases where the currents are conserved but not chiraly conserved leads in general to non-local actions, hence they are not very useful in those cases. A setting where they are still useful is in the case of $(1,1)$-supersymmetric WZW-models [15] 16] [17]. We will not spell out the details here because the results are exactly the same as in the bosonic case [8] except that fields are replaced by $(1,1)$-superfields, and that the shift

\footnotetext{
${ }^{6}$ In checking that the total result is equivalent to Buscher's transformations, one needs to use the fact that $\omega^{2}=\frac{1}{k^{2}}+g^{i j} k_{i} k_{j} / k^{4}$.
} 
of level $k \rightarrow k+c_{H}\left(c_{H}\right.$ is the dual Coxeter number of the subgroup $\left.H\right)$ does not take place due to cancellations between bosonic and fermionic determinants. Apart from these cases a satisfactory treatment of non-abelian duality in non-chiral cases in the functional integral or Hamiltonian approaches still remains elusive.

\section{Conclusions}

We have presented in this paper a canonical approach to (target space) duality in String Theory. We believe that this is a "minimal" approach in the sense that no extraneous structure needs to be introduced, and all standard results in the abelian case (and more) are easily recovered using it. In particular, the behavior of currents not commuting with those used to implement duality is clarified. All the generators of the full duality group $O(d, d, Z)$ can be described in terms of canonical transformations. This gives the impression that the duality group should be understood in terms of global symplectic diffeomorphisms. The configuration space of the two-dimensional field theories considered is the loop space of a manifold with isometries, the phase space is the cotangent space to this loop space. It would be useful to formulate the notion of duality in the context of some analogue of the group of disconnected diffeomorphisms, but for the time being we have not found such a construction. Finally non-abelian duality seems to fall beyond the scope of the Hamiltonian point of view advocated in this paper. Whether this is just a question of technical ingenuity or whether it is due to some conceptual difficulty in the very notion of non-abelian duality remains for the time being undecided.

\section{Acknowledgements}

We would like to thank E. Kiritsis and M.A. Vázquez-Mozo for useful discussions. E.A. and Y.L. were supported in part by the CICYT grant AEN 93/673 (Spain) and by a fellowship of Comunidad Autónoma de Madrid (YL).

\section{Note Added}

After completion of this work we learned that in reference 21] a canonical transformation was built relating the $S U(2)$ principal chiral model and its non-abelian dual with respect to the left action of the whole group. We thank T. Curtright and C. Zachos for bringing this information to our attention and for other discussions. We also learned that this non-abelian dual model was studied in [22]. 


\section{References}

[1] K. Kikkawa and M. Yamasaki, Phys. Lett. B149 (1984) 357; N. Sakai and I. Senda, Prog. Theor. Phys. 75 (1984) 692; L. Brink, M.B. Green and J.H. Schwarz, Nucl. Phys. B198 (1982) 474.

[2] T.H. Buscher, Phys. Lett. B194 (1987) 51, B201 (1988) 466.

[3] E. Álvarez and M.A.R. Osorio, Phys. Rev. D40 (1989) 1150.

[4] M. Roček and E. Verlinde, Nucl. Phys. B373 (1992) 630; A. Giveon and M. Roček, Nucl. Phys. B380 (1992) 128; A. Giveon and E. Kiritsis, Nucl. Phys. B411 (1994) 487; A. Giveon, M. Porrati and E. Rabinovici, Target Space Duality in String Theory, NYU-TH-94/01/01.

[5] E. Kiritsis, Nucl. Phys. B405 (1993) 109.

[6] E. Álvarez, L. Álvarez-Gaumé, J.L.F. Barbón and Y. Lozano, Nucl. Phys. B415 (1994) 71.

[7] X. de la Ossa and F. Quevedo, Nucl. Phys. B403 (1993) 377; A. Giveon and M. Roček, On Nonabelian Duality, ITP-SB-93-44, hep-th/9308154; M. Gasperini, R. Ricci and G. Veneziano, Phys. Lett. B319 (1993) 438.

[8] E. Alvarez, L. Alvarez-Gaumé and Y. Lozano, On Non-abelian Duality, CERN-TH 7204/94, hep-th/9403155. To appear in Nucl. Phys. B.

[9] A. Giveon, E. Rabinovici and G. Veneziano, Nucl. Phys. B322 (1989) 167; K.A. Meissner and G. Veneziano, Phys. Lett. B267 (1991) 33.

[10] G.I. Ghandour, Phys. Rev. D35 (1987) 1289; T. Curtright, Differential Geometrical Methods in Theoretical Physics: Physics and Geometry, ed. L.L. Chau and W. Nahm, Plenum, New York, (1990) 279; T. Curtright and G. Ghandour, Using Functional Methods to compute Quantum Effects in the Liouville Theory, proceedings of NATO Advanced Workshop, Coral Gables, FL, Jan 1991; and references therein.

[11] A. Anderson, Phys. Lett. B305 (1993) 67; A.Y. Shiekh, J. Math. Phys. 29 (1988) 913.

[12] Canonical transformations have also been used in String Theory in: J. Maharana and G. Veneziano, Nucl. Phys. B283 (1987) 126; J. Maharana and S. Mukherji, Phys. Lett. B284 (1992) 36.

[13] E. Witten, Comm. Math. Phys. 92 (1984) 455.

[14] A.M. Polyakov, P.B. Wiegmann, Phys. Lett. B131 (1983) 121, B141 (1984) 223.

[15] P. Di Vecchia, V. Knizhnik, J. Peterson and P. Rossi, Nucl. Phys. B253 (1985) 701.

[16] A. Giveon, E. Rabinovici and A.A. Tseytlin, Nucl. Phys. B409 (1993) 339. 
[17] A. Das and J. Maharana, Duality of the Superstring in Superspace, UR-1338, hepth/9401147.

[18] I. Bars and K. Sfetsos, Mod. Phys. Lett. A7 (1992) 1091.

[19] I. Antoniadis and N. Obers, Plane Gravitational Waves in String Theory, CPTHA299.0494, hep-th/9403191.

[20] E. Kiritsis and N. Obers, A New Duality Symmetry in String Theory, CERN-TH $7310 / 94$, hep-th/9406082.

[21] T. Curtright and C. Zachos, Phys. Rev. D49 (1994) 5408.

[22] B.E. Fridling and A. Jevicki, Phys. Lett. B134 (1984) 70. 